\title{
APPLICATION OF A GENERIC SIMULATION MODEL TO OPTIMIZE PRODUCTION AND WORKFORCE PLANNING AT AN AUTOMOTIVE SUPPLIER
}

\author{
Klaus Altendorfer \\ Thomas Felberbauer \\ Daniel Gruber \\ Alexander Hübl \\ University of Applied Sciences Upper Austria \\ Wehrgrabengasse 1-3 \\ A-4400 Steyr, AUSTRIA
}

\begin{abstract}
This paper presents a comprehensive simulation project in the area of an automotive supplier. The company produces car styling serial and original accessory parts made from plastic for internal and external applications in passenger cars. For the foaming division, which is identified as the bottleneck, different personnel and qualification scenarios, set-up optimizations and lot-sizing strategies are compared with the current situation. Key performance measures reported are inventory, tardiness and service level. The changes in organizational costs (e.g. employee training, additional employees, etc.), due to the scenarios, are not considered and are traded off with the logistical potential by the company itself. Results of the simulation study indicate that a combination of an additional fitter during night shift, minor reductions of set-up times and reduced lot-sizes leads to an inventory reduction of $\sim 10.6 \%$ and a service level improvement of $\sim 8 \%$ compared to the current situation.
\end{abstract}

\section{INTRODUCTION}

The automotive industry in Austria is an important sector for the Austrian economy. According to the Austrian Economic Chambers (http://wko.at/fahrzeuge/default.htm) approximately 30,000 employees generate a volume of production of 12,500 billion Euros. Most of these companies are suppliers in the automotive supply chain. The investigated company is one of these suppliers and results of this study indicate the potential for improvement by applying simulation; this also holds true for other companies in this field.

A wide range of publications concerning MRP (material requirements planning) as planning a method are available showing that MRP is applied in industry and intensively studied (Weeks 1981; Yano 1987; Gong et al. 1994; Elhafsi 2002; Axsäter 2005; Altendorfer and Minner 2011; Hopp and Spearman 2008). MRP can handle any kind of complexity and stochastic behavior in customer demand and processing time, which supports its enormous popularity among industry (see Chang and Yih (1994) and Hopp and Spearman (2008)). Among others, one of the main parameters for MRP is the lot-sizing decision influencing inventory, set-up effort (and therefore utilization) and service level (Hopp and Spearman 2008, Jodlbauer and Huber 2008). First publications in this field were Wagner and Whitin (1958) and Manne (1958) for dynamic but deterministic customer demand in a rather streamlined setting. Recent work on lot-sizing discusses, for example, customer demand variance (Ho 1995), solution algorithms (Dellaert and Jeunet 2000), set-up influence (Gardiner and Blackstone 1995) and simulation for lot-size optimization (Jodlbauer and Huber 2008). In this study, simulation is also applied to identify the influence of lot-sizes in the investigated production system. 
Another factor influencing the logistical performance of a production system is the workforce availability and qualification for different tasks. Mazzola et al. (1998) explore a multiproduct production planning problem, whereby the work-force productivity reflects a learning-curve effect. The authors show that this problem is NP hard and provide a branch-and-bound algorithm and a tabu-search heuristic to solve this problem. Carrillo and Gaimon (2000) demonstrate in their optimal control model that companies should invest in skills of employees and process changes to be more effective or less expensive. Thorwarth et al. (2009) investigate a flexible workload allocation for hospital staff. Stratman et al. (2004) develope a simulation model for analyzing worker skill dynamics whereby the use of temporary versus permanent workers on manufacturing costs is investigated. In their model they have implemented short product life cycles, mid-volume production quantities and a production environment consisting of assembly, inspection and testing.

In this paper we examine the foaming division of an Austrian automotive supplier. The production planning and control method for the materials is MRP. The sales plan of the last business year is input for all investigated simulation scenarios. The validation process is twofold. On the one hand, performance measures from the simulation model (inventory, work in process (WIP) and service level) are compared with the ones of the last business year. On the other hand, we conduct in depth analysis of the simulation model with the company partners. The production area has three production stages (foaming, manual trimming one and manual trimming two). The target of the simulation study is to compare different personnel and qualification scenarios, set-up optimizations and lot-sizing policies with the past business year and provide managerial insights. As sketched in the review above, there are analytical models for all of these decisions available, however, simulation is a convenient tool to handle the complexity of the combination of these decisions and is therefore applied in this study.

\section{MODEL}

For the simulation project, a generic simulation model is used, similar to the simulation study in Felberbauer, Altendorder and Hübl (2012). The core concept of the generic simulation model is presented in Hübl et al. (2011) which explains the parameterization by a database. Thereby, it is possible to define different simulation scenarios without any adaption of the simulation model itself. The relational database model is designed according to the relevant simulation data for production planning simulation. Generally, the simulation model imports the data from the database in an initial phase. On the one hand, the data is used at the startup of the simulation model to replicate the single simulation modules and thereby creating the production system structure. On the other hand, the data is used to initialize the random number generators for processing, set-up and repair times, as well as to parameterize the production planning. Basically, the database distinguishes between master data and transaction data. The master data defines the structure of the production system and consists of:

- bill of material (BOM): defining the relationship between parent and child item(s)

- routings: definition of the machine groups and their machines, including the capacities

- production planning parameters for each item

- $\quad$ shift calendar for all skill groups, including holidays

- definition of skill groups of the employees, including their capacities

The transaction data is characterized by distributions, which are used for generating the random variables in the model. The distribution parameters are defined in tables of the database. A distribution is implemented for processing time, set-up-time, mean time to repair and mean time between failure, customer demand, customer required lead time and replenishment lead time. The simulation time (model run time) is also customized in the database.

The different modules of the scalable simulation model itself are programmed in Anylogic 6.8. The simulation model consists of the five modules: customer, production planning, material release, resources 
and analysis. For a detailed description of the simulation modules compare, Felberbauer, Altendorder, and Hübl (2012) and Hübl et al. (2011).

\section{PROBLEM DESCRIPTION}

The flow shop investigated consists of three different areas. The first area is the foaming division containing three different machines (FT1, FT2 and FT3) which are technologically not identical. The second (manual trimming one) and third (manual trimming two) area are hand-work places where the foamed products are refinished. According to a capacity analysis of the last business year, the foaming machines are the bottleneck of this production system.

For simulation time and complexity reasons, an $\mathrm{ABC}$-analysis for capacity consumption is conducted according to the past sales plan. Based on this analysis, 36 finished products (out of 230) are identified which account for $80 \%$ of the annual capacity consumption. For "A"-finished products and their sub items the production planning is simulated in detail. For " $\mathrm{B}$ " and " $\mathrm{C}$ "-articles, only their capacity consumption but not their production planning, order generation and dispatching is simulated. This approach reduces the simulation time and the data acquisition effort, e.g. for planning parameterization, bill of material, routing information, etc.

The processing times, set-up times, mean times between failure and mean time to repair are modeled as independent lognormal distributed random variables and their expected value and coefficient of variation are extracted from the MES-system (manufacturing execution system) of the company. At stations trimming one and trimming two, no set-up is needed. At the foaming machines, a set-up is needed between orders for different materials, whereby its mean and coefficient of variation are independent of the materials to process. All materials are MRP planned and the MRP run is conducted in ERP-system (enterprise resource planning) SAP R3 once a day. The foaming division is a pure make-to-order (MTO) system, therefore no forecast information is considered in the MRP-run.

For the investigated area, two different shift systems are applied. The production employees working in the foaming, trimming one and trimming two divisions, labor five days a week in three shifts (shift one from 6:00 am-2:00 pm, shift two from 2:00 pm - 10:00 pm and shift three from 10:00 pm- 6:00 am). The set up team (fitters) which is responsible for the set-ups in the foaming division (machines: FT1, FT2 and FT3) works five days a week in two shifts (shift one form 6:00 am-2:00 pm and shift two from 2:00 pm $10: 00 \mathrm{pm}$ ). If the planned production order amount is reached during the third shift and there is no production order with the same product waiting, the production employee in the foaming division produces until the set-up team is available in the first shift at 6:00 am. This policy leads to an overproduction in comparison to the planned order amount and is only possible due to the fact that the raw material is infinitely available. The prioritization of work intensive orders (work content $>10 \mathrm{~h}$ ) before the shift end of the second shift is one action defined by the management to dampen problems caused by this policy.

From the practical as well as the research perspective, an interesting question is how this overproduction policy influences the logistical performance measures inventory and service level. In detail, management claimed a low service level performance of this production system and provided a set of possible measures to improve the situation. Such measures are employing fitters also in the night shift, qualify workers to conduct the set-up process during night shift, stop production when production amount is reached, reduce set-up-times by implementing technical improvements and reduce the production lot-size. Therefore, a set of scenarios is identified in cooperation with the management and simulation is applied to evaluate the key performance indicators inventory, tardiness and service level for the latter explained scenarios. 


\section{RESULTS}

\subsection{Experiment Design}

In the four scenarios tested (S1.x, S2.x, S3.x, S4.x) the customer demand per month and year is always kept equal in order to have the same amount of products produced within the simulation time. In S1, the possibility of setting up machines in night shift is evaluated. This can be achieved either by employing an additional fitter for the night shift (S1.1) or by training a worker to be able to accomplish the set-up process (S1.2). In S2, set-up- time reduction is tested. In the first case (S2.1), set-up-times reduce from 30 to 20 minutes on average, which can be achieved with little effort by the company. The second case (S2.2) implies a major change in the set-up process and the average set-up-time is reduced to eight minutes. In S3 the lot-size is set to $50 \%$ of current value with a minimum of 200 pieces per production order, which is a feasible policy according to the technical restrictions of the production system. Scenario S4 comprises a combination of reduced set-up-times and the new lot-sizing policy.

The performance-measures are service level [\%], tardiness in days [d], utilization of the three machine groups FT1-FT3 [\%], inventory on storage measured in pieces[pcs.) and inventory plus work in process [pcs.]. In the experiments, five years with 365 days each, are analyzed. To reach a steady state of the simulation model one year, representing the warm up phase is removed from the analysis. To account for the stochastic behavior of the simulation model the $99 \%$ confidence interval $( \pm \mathrm{CI})$ is quoted for each key performance measure. Each run is replicated 100 times. Performance measures are evaluated for each scenario and compared with current situation (S0) of the company.

\subsection{S0 - Current Situation}

All necessary data for the generic simulation model database is identified from current values of the company. Additionally, safety stocks had to be implemented in order to meet the average inventory of products of year 2011 in the simulation results. This is necessary to convert human interactions of production planning into a logical simulation rule, as production planning is often turning planned orders into production orders manually. The result shown in Table 1 represents the results of trading year 2011 for the investigated production system. As mentioned above, the simulation results have been validated with company experts.

Table 1: Performance measures for the current situation S0.

\begin{tabular}{|c|c|c|c|}
\hline KPI & Unit & SO & $\mathbf{\pm C I ~ S 0 ~}$ \\
\hline Service level & $\%$ & 70 & 0.24 \\
\hline Avg. Tardiness & $\mathrm{d}$ & 0.4 & 0.03 \\
\hline Utilization FT1 & $\%$ & 82 & 0.11 \\
\hline Utilization FT2 & $\%$ & 84 & 0.11 \\
\hline Utilization FT3 & $\%$ & 83 & 0.13 \\
\hline Avg. Inventory & Pcs. & 8,770 & 26 \\
\hline Avg. Inventory + WIP & Pcs. & 9,940 & 25 \\
\hline
\end{tabular}

Table 1 shows a service level after the process step "trimming 2 " of $70 \%$. This means only 7 out of 10 production orders of this process step are finished in time. The average inventory is 8,770 pieces over all three investigated process steps whereas inventory including WIP is around 9,940 pieces. The utilization of machines 1 to 3 in the bottleneck-area is visualized to show that the production program is equal in all scenarios tested. 


\subsection{S1 - Availability of Setting Up Machines During Night Shift}

In $\mathrm{S} 1$, the possibility of setting up machines during, night shift is tested. This is possible in two different ways, either an additional fitter is employed for the night shift (S1.1) or an existing worker is trained to be capable of doing the set-up tasks (S1.2). In scenario S1.2, the set-up time is increased by $50 \%$ as the qualified worker is doing the set-up process alone and the set-up process is, therefore, not as quick as a fitter who has a worker to help during set-up. The result presented in Table 2 shows the two scenarios in comparison to the current situation S0.

Table 2: Performance measures for S1 - availability of setting up machines during night shift.

\begin{tabular}{|c|c|c|c|c|c|c|c|}
\hline KPI & Unit & S0 & \pm CI S0 & S1.1 & \pm Cl S1.1 & S1.2 & \pm CI S1.2 \\
\hline Service level & $\%$ & 70 & 0.24 & 71 & 0.20 & 68 & 0.26 \\
\hline Avg. Tardiness & d & 0.4 & 0.03 & 0.3 & 0.02 & 0.4 & 0.05 \\
\hline Utilization FT1 & $\%$ & 82 & 0.11 & 83 & 0.12 & 83 & 0.13 \\
\hline Utilization FT2 & $\%$ & 84 & 0.11 & 85 & 0.13 & 85 & 0.19 \\
\hline Utilization FT3 & $\%$ & 83 & 0.13 & 83 & 0.14 & 83 & 0.12 \\
\hline Avg. Inventory & Pcs. & 8,770 & 26 & 7,400 & 10 & 7,250 & 13 \\
\hline Avg. Inventory + WIP & Pcs. & 9,940 & 25 & 8,530 & 10 & 8,380 & 12 \\
\hline
\end{tabular}

The decrease in inventory and inventory plus WIP is reasonable due to the increased flexibility in setting up machines during night shift, where only the needed amount of products is actually produced. Remarkable is the fact, that the service level is nearly not influenced by this measure. Therefore, both of these measures reduce in fact overproduction and therefore inventory. However, the provided service level does not improve. One explanation for this could be that lot-sizes and planned lead-times are not set accurately in the current situation.

\subsection{S2 - Reduced Set-Up Times}

In S2, the current situation is tested with reduced set-up times. Again, two possible changes are tested; in the first case (S2.1, S2.3) set-up times are reduced to 20 minutes on average with a standard deviation of $+/-5$ minutes. This reduced set-up-time was defined by the company and could be implemented with minor changes in the set-up process, such as increasing the amount of external set-up and further training of fitter personnel. In the second case (S2.2, S2.4), set-up-times are reduced to 8 minutes on average with a standard deviation of $+/-1$ minute. For this reduction, a major change in the set-up process is necessary, i.e. new crane for changing tools. Both scenarios of $\mathrm{S} 2$ are tested for the current situation of the company (S2.1, S2.2) and also for the possibility of setting up machines during night shift (S2.3, S2.4) with an additional fitter. The results of S2 are presented in Table 3:

Table 3: Performance measures for S2 - reduced set-up-times.

\begin{tabular}{|c|c|c|c|c|c|c|c|c|c|c|c|}
\hline KPI & Unit & S0 & \pm CI S0 & S2.1 & \pm CI S2.1 & S2.2 & \pm CI S2.2 & S2.3 & \pm CI S2.3 & S2.4 & \pm CI S2.4 \\
\hline Service level & $\%$ & 70 & 0.24 & 73 & 0.19 & 74 & 0.11 & 73 & 0.15 & 74 & 0.13 \\
\hline Avg. Tardiness & d & 0.4 & 0.03 & 0.3 & 0.02 & 0.2 & 0.01 & 0.3 & 0.02 & 0.3 & 0.02 \\
\hline Utilization FT1 & $\%$ & 82 & 0.11 & 80 & 0.12 & 78 & 0.11 & 80 & 0.13 & 77 & 0.16 \\
\hline Utilization FT2 & $\%$ & 84 & 0.11 & 82 & 0.11 & 80 & 0.14 & 82 & 0.16 & 80 & 0.18 \\
\hline Utilization FT3 & $\%$ & 83 & 0.13 & 80 & 0.14 & 78 & 0.14 & 80 & 0.15 & 78 & 0.18 \\
\hline Avg. Inventory & Pcs. & 8,770 & 26 & 8,920 & 23 & 8,980 & 22 & 7,560 & 7 & 7,650 & 6 \\
\hline Avg. Inventory + WIP & Pcs. & 9,940 & 25 & 10,080 & 23 & 10,130 & 22 & 8,690 & 8 & 8,790 & 7 \\
\hline
\end{tabular}


For the current situation of the company, a slight improvement in service level can be achieved through reduced set-up times (S2.1-S2.4). In terms of service level, it seems like there is no difference between the current situation (S2.1, S2.2) and the possibility of setting up machines during night shifts (S2.3, S2.4). Again, it is conjectured that this effect occurs due to non-optimal settings of current planning parameters. The increase in inventory and inventory plus WIP in S2.1 and S2.2 can be described due to the reduction of production lead-time and therefore longer average waiting times in finished goods inventory. The decrease in inventory in S2.3 and S2.4 in comparison to S2.1 and S2.2, which is linked to setups in the night shift, is $\sim 15.2 \%$ and $\sim 14.8 \%$ respectively.

\subsection{S3 - Halved Lot-Sizes}

In Scenario S3 the current situation (S3.1) and situation with the possibility to setting-up machines during night shift with an additional fitter (S3.2) is tested with the new lot-sizing policy introduced above (half of the current lot-size with a minimum of 200 pieces per order).

Table 4: Performance measures for S3 - halved lot-sizes.

\begin{tabular}{|c|c|c|c|c|c|c|c|}
\hline KPI & Unit & S0 & \pm CI S0 & S3.1 & \pm CI S3.1 & S3.2 & \pm CI S3.2 \\
\hline Service level & $\%$ & 70 & 0.24 & 75 & 0.26 & 75 & 0.24 \\
\hline Avg. Tardiness & d & 0.4 & 0.03 & 0.3 & 0.04 & 0.3 & 0.04 \\
\hline Utilization FT1 & $\%$ & 82 & 0.11 & 83 & 0.09 & 84 & 0.18 \\
\hline Utilization FT2 & $\%$ & 84 & 0.11 & 84 & 0.10 & 86 & 0.19 \\
\hline Utilization FT3 & $\%$ & 83 & 0.13 & 84 & 0.11 & 84 & 0.20 \\
\hline Avg. Inventory & Pcs. & 8,770 & 26 & 9,140 & 25 & 7,610 & 13 \\
\hline Avg. Inventory + WIP & Pcs. & 9,940 & 25 & 10,250 & 25 & 8,700 & 14 \\
\hline
\end{tabular}

The results for S3.1 in Table 4 show a significant increase of service level (5\%) and a slight increase in average inventory which is again linked to an increase in the finished goods inventory. Remarkable is the fact, that with an additional fitter during night shift (S3.2), the service level stays unchanged but the average inventory can be reduced by $\sim 16.7 \%$ compared to $\mathrm{S} 3.1$.

\subsection{S4 - Combination of S2 and S3}

In Scenario 4, a combination of reduced set-up times (S2) and new lot-sizing policy (S3) is tested for the current situation and the case with an additional fitter in night shift. In S4.1 (without fitter in night shift) and S4.2 (with fitter in night shift) the reduced set-up-time of 20 minutes on average is applied. In S4.3 (without fitter in night shift) and S4.4 (with fitter in night shift) the reduced set-up-time of 8 minutes on average is tested. The results of S4 are presented in Table 5:

Table 5: Performance measures for S4 - reduced set-up-times and halved lot-sizes.

\begin{tabular}{|c|c|c|c|c|c|c|c|c|c|c|c|}
\hline KPI & Unit & S0 & \pm CI S0 & S4.1 & \pm CI S4.1 & S4.2 & \pm CI S4.2 & S4.3 & \pm CI S4.3 & S4.4 & \pm CI S4.4 \\
\hline Service level & $\%$ & 70 & 0.24 & 79 & 0.17 & 78 & 0.17 & 80 & 0.12 & 79 & 0.13 \\
\hline Avg. Tardiness & d & 0.4 & 0.03 & 0.2 & 0.02 & 0.2 & 0.03 & 0.1 & 0.02 & 0.2 & 0.04 \\
\hline Utilization FT1 & $\%$ & 82 & 0.11 & 80 & 0.11 & 81 & 0.22 & 78 & 0.12 & 78 & 0.22 \\
\hline Utilization FT2 & $\%$ & 84 & 0.11 & 82 & 0.11 & 83 & 0.21 & 80 & 0.12 & 81 & 0.21 \\
\hline Utilization FT3 & $\%$ & 83 & 0.13 & 81 & 0.13 & 81 & 0.24 & 78 & 0.13 & 78 & 0.25 \\
\hline Avg. Inventory & Pcs. & 8,770 & 26 & 9,330 & 24 & 7,840 & 10 & 9,430 & 18 & 7,960 & 9 \\
\hline Avg. Inventory + WIP & Pcs. & 9,940 & 25 & 10,430 & 24 & 8,930 & 11 & 10,530 & 18 & 9,050 & 10 \\
\hline
\end{tabular}


Looking at the results from Table 5 shows that the combination of applying the new lot-sizing policy, reducing set-up-time and providing an additional fitter in the night shift leads to the best results. The service level can be increased up to $\sim 80 \%$, which means an increase by $\sim 10 \%$. The slight reduction in utilization is due to less overall set-up-times. Note that an investment in reducing the set-up process is only efficient if also the lot-size is adjusted. Since no cost information on providing a fitter for the night shift and for reducing the set-up-time is available, it depends on the company to decide which scenario is the best. However, assuming that a decrease in set-up-time to 20 minutes and applying an additional fitter are possible with reasonable costs, indicates that scenario S4.2 can probably be selected as the best scenario. In this scenario, the service level is increased by $8 \%$ up to $\sim 78 \%$ and the average inventory can be reduced by $10.6 \%$ in comparison to the current situation. Additionally, the average delay is reduced to 0.2 days per order.

\section{CONCLUSION}

The simulation study performed in this paper shows that improvement measures identified in collaboration with leading personnel of the studied company can lead to a significant improvement of the production system. An additional fitter during the night shift reduces inventory by $\sim 16 \%$ with a steady service level. Minor changes in the set-up process outweigh major changes with implementation costs in the case of investigated key performance indicators. A new lot-sizing policy with reduced lot-sizes shows that the service level can be improved significant by 7\%, regardless of employing an additional fitter during night shift or not. Applying a combination of the evaluated improvement measures (additional fitter during night shift, minor reduction of set-up-time, new lot-sizing policy) leads to an inventory reduction of $\sim 10.6 \%$ and a service level improvement of $\sim 8 \%$ in comparison to the current situation.

Even though this study shows some practically relevant results which lead to an improved performance of the studied production system it also has some mayor limitations. To identify the whole improvement potential for the investigated production system, the MRP planning parameters lot-size, planned lead time and safety stock would have to be systematically analyzed by parameter variation experiments or by applying certain optimization heuristics. This is left for further research and will probably be conducted in a further simulation project with the company.

\section{ACKNOWLEDGMENTS}

This paper was written within the framework of the project "SimGen" (project number 826789) funded by the Austrian Research Promotion Agency (FFG).

\section{REFERENCES}

Altendorfer, K., and S. Minner. 2011. "Simultaneous Optimization of Capacity and Planned Lead Time in a Two-Stage Production System with Different Customer Due Dates." European Journal of Operational Research 213: 134-146.

Axsäter, S. 2005. "Planning Order Releases for an Assembly System with Random Operation Times." OR Spectrum 27: 459-470.

Carrillo, J., and C. Gaimon. 2000. "Improving Manufacturing Performance Through Process Change and Knowledge Creation." Management Science 46: 265-288.

Chang, M., and Y. Yih. 1994. "Generic Kanban Systems for Dynamic Environments." International Journal of Production Research 32: 889-902.

Dellaert, N., and J. Jeunet. 2000. "Solving Large Unconstrained Multilevel Lot-Sizing Problems Using a Hybrid Genetic Algorithm.” International Journal of Production Research 38: 1083-1099.

Elhafsi, M. 2002. "Optimal Leadtimes Planning in Serial Production Systems with Earliness and Tardiness Costs." IIE Transactions 34: 233-243. 
Felberbauer, T., K. Altendorfer, and A. Hübl. 2012. „Using a Scalable Simulation Model to Evaluate the Performance of Production System Segmentation in a Combined MRP and Kanban System." In Proceedings of the 2012 Winter Simulation Conference, edited by C. Laroque, J. Himmelspach, R. Pasupathy, O. Rose, and A.M. Uhrmacher, 1-12. 1570. Piscataway, New Jersey: Institute of Electrical and Electronics Engineers, Inc.

Gardiner, S., and J. Blackstone. 1995. "Setups and Effective Capacity: The Impact of Lot-Sizing Techniques in an MRP Environment." Production Planning \& Control 6: 26-38.

Gong, L., T. Kok., and J. Ding. 1994. "Optimal Leadtimes Planning in a Serial Production System.” Management Science 40: 629-632.

Ho, C. 1995. "Examining the Impact of Demand Lumpiness of the Lot-Sizing Performance in MRP Systems." International Journal of Production Research 33: 2579-2599.

Hopp, W. J., and M. L. Spearman. 2008. Factory Physics. Boston: Mc Graw Hill / Irwin.

Hübl, A., K. Altendorfer, H. Jodlbauer, M. Gansterer, and R. F. Hartl. 2011. Flexible Model for Analyzing Production Systems with Discrete Event Simulation." In Proceedings of the 2011 Winter Simulation Conference, edited by S. Jain, R.R. Creasey, J.Himmelspach, K.P. White, and M. Fu, 1559-1570. Piscataway, New Jersey: Institute of Electrical and Electronics Engineers, Inc.

Jodlbauer, H., and A. Huber. 2008. "Service-Level Performance of MRP, Kanban, CONWIP and DBR due to Parameter Stability and Environmental Robustness." International Journal of Production Research 46: 2179-2195.

Manne, S. 1958. "Programming of Economic Lot Sizes." Management Science 4: 115-135.

Mazzola, B., W. Neebe, and M. Rump. 1998. "Multiproduct Production Planning in the Presence of Work-Force Learning.” European Journal of Operational Research 106: 336-356.

Stratman, K., V. Roth, and G. Gilland. 2004. "The Deployment of Temporary Production Workers in Assembly Operations: A Case Study of the Hidden Costs of Learning and Forgetting." Journal of Operations Management 21: 689-707.

Thorwarth, M., A. Arisha, and P. Harper. 2009. "Simulation Model to Investigate Flexible Workload Management for healthcare and Servicescape Environment." In Proceedings of the 2009 Winter Simulation Conference, edited by M. D. Rossetti, R. R. Hill, B. Johansson, A. Dunkin and R. G. Ingalls, 1946-1956. 1570. Piscataway, New Jersey: Institute of Electrical and Electronics Engineers, Inc.

Wagner, H., and T. Whitin. 1958. "Dynamic Version of the Economic Lot Size." Management Science 5: 89-96.

Weeks, J. K. 1981. “Optimizing Planned Lead Times and Delivery Dates.” 21st Annual Conference Proceeding, American Production and Inventory Society 177-188.

Yano, C. A. 1987. "Setting Planned Leadtimes in Serial Production Systems with Tardiness Costs." Management Science 33: 95-106.

\section{AUTHOR BIOGRAPHIES}

KLAUS ALTENDORFER works as a Professor in the field of Operations Management at the University of Applied Sciences, Steyr (Austria). He received his $\mathrm{PhD}$ degree in logistics and operations management with distinction and has professional experience in stochastic inventory models and production planning and control. His e-mail address is klaus.altendorfer@fh-steyr.at.

THOMAS FELBERBAUER works as a Research Associate in the field of Operations Management at the University of Applied Sciences, Steyr (Austria). His research interests are discrete event simulation and combinatorial optimization. His email address is thomas.felberbauer@fh-steyr.at.

DANIEL GRUBER works in the planning department at voestalpine, Linz (Austria). His email address is daniel.gruber@voestalpine.com. 


\section{Altendorfer, Felberbauer, Gruber, and Hübl}

ALEXANDER HÜBL works as a Research Associate in the field of Operations Management at the University of Applied Sciences, Steyr (Austria). His research interests are discrete event simulation and quantitative methods for capacity setting. His email address is alexander.huebl@fh-steyr.at. 